\title{
Corela
}

Cognition, représentation, langage

HS-16 | 2015

Diversité des pratiques de recherche en science du langage

\section{Les injonctifs averbaux (sans verbe conjugué)}

D'une approche en langue à une analyse de corpus

\section{Chinh Nguyen Minh}

\section{(2) OpenEdition}

\section{Journals}

Édition électronique

URL : http://journals.openedition.org/corela/3759

DOI : $10.4000 /$ corela.3759

ISSN : $1638-573 \mathrm{X}$

Éditeur

Cercle linguistique du Centre et de l'Ouest - CerLICO

Référence électronique

Chinh Nguyen Minh, «Les injonctifs averbaux (sans verbe conjugué) », Corela [En ligne], HS-16 | 2015, mis en ligne le 04 juin 2015, consulté le 19 avril 2019. URL : http://journals.openedition.org/ corela/3759 ; DOI : 10.4000/corela.3759

Ce document a été généré automatiquement le 19 avril 2019

\section{(c) (1)(2)}

Corela - cognition, représentation, langage est mis à disposition selon les termes de la licence Creative Commons Attribution - Pas d'Utilisation Commerciale - Partage dans les Mêmes Conditions 4.0 International. 


\title{
Les injonctifs averbaux (sans verbe conjugué)
}

D’une approche en langue à une analyse de corpus

\author{
Chinh Nguyen Minh
}

1 Dans les recherches récentes sur l'énoncé averbal, les relations entre les éléments explicites et implicites sont bien étudiées. Cependant, la valeur injonctive que les énoncés averbaux peuvent exprimer a peu été abordée. Ainsi, Lefeuvre (2003) a abordé la modalité injonctive des énoncés averbaux mais elle n'est pas entrée dans les détails. Je propose donc dans cet article une description linguistique et pragmatique des énoncés averbaux à valeur injonctive et une description des compétences mobilisées dans différentes situations sociales, autrement dit, la répartition de ces formes en fonction des différents genres de discours.

2 Je présente les fondements théoriques sur lesquels je définis mon objet d'étude dans la première section de cet article, la méthodologie et le corpus d'étude dans la deuxième section. La troisième section est réservée à une analyse syntaxique, sémantique et pragmatique des injonctifs averbaux. La quatrième section est consacrée à la description des fréquences d'emploi des injonctifs averbaux dans les sous-corpus.

\section{Définitions}

\subsection{De l'injonction}

3 L'acte injonctif (l'acte directif) est classé par Searle (1979, $1990: 358-360)$ comme une catégorie indépendante dans sa liste de cinq actes illocutoires. L'acte injonctif est un acte dont le but illocutoire est de pousser l'interlocuteur (qui est parfois le locuteur lui-même) à la réalisation d'une action future. Le locuteur essaie de faire faire les choses. L'élément définitoire essentiel est ce but illocutoire de l'acte injonctif : chercher à rendre le monde conforme au contenu propositionnel (qui contient l'action future de l'interlocuteur). L'acte injonctif exprime les désirs, la volonté et l'intention du locuteur. Les conditions de sincérité sont donc les désirs, les souhaits et la volonté du locuteur. Les actes de ce type 
varient selon le degré d'effort déployé par le locuteur afin d'atteindre son but, du plus modeste (invitation, proposition ou suggestion) au plus vigoureux (insistance ou prière) ou péremptoire (ordre, autorisation ou commandement). Searle indique dans cette catégorie les actes associés aux verbes ordonner, commander, demander, requérir, prier, solliciter, supplier, ainsi que permettre, conseiller, inviter, etc. (Searle, 1990 : 359-360).

Par nature, l'acte injonctif accompagne l'intention d'agir sur autrui pour obtenir l'accomplissement de l'action future exprimée dans le contenu propositionnel. Le locuteur doit alors fournir à l'allocutaire une raison pour réaliser l'action en produisant l'énoncé. Autrement dit, l'allocutaire doit reconnaitre au moins une raison impliquée dans le contenu propositionnel de l'injonction pour laquelle il réalise l'action demandée. Cependant, par l'influence des éléments linguistiques et extra-linguistiques, cette raison est parfois à déduire du contexte.

\subsection{De l'énoncé averbal}

5 Formellement, l'énoncé averbal ne comporte ni verbe ni copule (Benveniste, 1966 : 151). S'opposant à la définition de l'énoncé verbal qui s'appuie sur la présence du verbe conjugué, les définitions de l'énoncé averbal insistent sur l'absence du verbe. L'énoncé averbal se définit donc « par opposition à une phrase verbale » (Riegel et al., $2009: 457$ ).

6 Toutefois, selon Le Goffic, l'absence du verbe «n'empêche pas la manifestation d'une modalité de phrase et d'un acte de discours » $(1993: 510)$ et ce sont la prosodie et l'ordre des mots qui permettent de prédiquer et d'asserter. Suivant cette idée, Lefeuvre propose la définition suivante de l'énoncé averbal, qui est:

[...] une structure syntaxique constituée d'un prédicat averbal et d'une modalité, selon deux possibilités. Le prédicat averbal est relié par la modalité, à un sujet explicite ou implicite. Ou bien, le prédicat est simplement posé par la modalité. Des éléments extra-prédicatifs peuvent apparaitre dans la constitution de la phrase averbale. (Lefeuvre, $2003: 29$ )

7 Selon ces définitions, les injonctifs averbaux comportent un prédicat sans verbe et une modalité injonctive. Le récepteur peut être explicite ou implicite. La référence au récepteur est assurée par des moyens de surface ou contextuels.

\subsubsection{Prédication}

Deulofeu (2004) distingue trois approches différentes. Celle de Riegel et al. (2009) qui traitent des énoncés averbaux comme des structures atypiques que l'on utilise dans des contextes particuliers ; celle de Bally (1944), Benveniste (1966), Hjelmslev (1971), Le Goffic (1993) et Lefeuvre (2003) qui ont montré qu'il s'agit d'énoncés normaux dont la structure syntaxique est spécifique. Enfin, celles de Meillet (1924), Perrot (1994), BlancheBenveniste (1997) et Cresti (1998) qui ont surtout insisté sur le fait que les énoncés averbaux s'intègrent à la grammaire de la langue et qu'ils peuvent être décrits comme les autres.

Normalement, le verbe assure la prédication et l'ancrage situationnel: temporel, personnel, aspectuel, etc. L'absence du verbe conjugué provoque certaines conséquences. Benveniste considère l'énoncé averbal comme « intemporel, impersonnel, non-modal» (1966 : 159). Comme lui, Le Goffic ne veut pas «parler de sujet : dans le type très usuel 
Délicieux, ce café!", puisqu'il n'y a pas d'accord avec un verbe. Il propose de parler de « thème postposé » plutôt que de sujet au sens strict. En revanche, adoptant une approche non-morphologique, il remarque qu'il faut interpréter l'énoncé averbal comme lié au présent (au sens sémantique) "même si ce présent peut s'étendre sans borne » et que l'énoncé averbal exprime toujours une modalité de phrase et un acte de discours (Le Goffic, 1993 : 510).

Riegel et al. estiment que l'énoncé averbal est «avant tout sensible aux variations de la situation d'énonciation particulière ", qu'il " ne prend sa valeur que dans une situation déterminée, où l'interprétation de l'énoncé dépend de la relation d'énonciation et du cadre spatio-temporel » (Riegel et al., 2009 : 457). Ils insistent donc sur le rôle du contexte linguistique pour l'interprétation, et, dans la plupart des cas, du contexte situationnel.

11 Certains linguistes ont signalé le risque de confondre les énoncés averbaux avec les énoncés elliptiques (Jesperson, [1924] 1971 : 437), tronqués (Le Goffic, 1993 : 510), ou avec les membres de phrase (Lefeuvre, 2003 : 69-70). Les énoncés qui dépendent de l'énoncé précédent ou suivant et dont le contexte permet de restituer la forme complète (le verbe notamment) sont considérés par ces auteurs comme elliptiques ou membres de phrase. Je ne tiendrai pas compte de cette différence pour recueillir les exemples. Cette distinction entre énoncé averbal ou elliptique, énoncé averbal ou membre de l'énoncé ne sera pas mobilisée. Tous les énoncés sans verbe conjugué en surface sont donc pris en compte. Selon ce point de vue, les énoncés qui n'ont formellement pas de verbe en surface sont averbaux, même si l'on peut restituer le verbe grâce au contexte linguistique ou situationnel. L'injonction est une action qui en appelle une autre. Les énoncés averbaux examinés doivent référer implicitement ou explicitement à une action. Cette référence doit être reconnue par le récepteur pour que l'action future soit réalisable. Sinon le locuteur reformule son injonction afin d'obtenir son but comme dans l'exemple suivant :

\begin{tabular}{|c|c|}
\hline BEA & café $\backslash$ \\
\hline BEA & barbara tu veux un café/= (Corpus TABACCO - CLAPI) \\
\hline
\end{tabular}

\subsubsection{Classement}

Pinchon, 1962 : 514-517 ; Riegel et al., 2009 : 458-460 ; Lefeuvre, 2003) ;

-à partir des classes de mots : nom, adjectif, adverbe, préposition (Tesnière, 1959) ;

-selon les termes et la valeur sémantique : attributif, locatif, existentiel (Le Goffic, 1993 : 509-523).

Suivant la méthode d'analyse des signifiés à partir de signifiants, j'adopte un classement qui regroupe les énoncés averbaux «autour d'une tête» (Le Goffic, 1993: 22) et qui propose quatre classes : substantif, adjectif, verbe et adverbe (Moignet, 1981: 13, Le Goffic, 1993 : 20). Lefeuvre (2003 : 57) y ajoute le pronom et la préposition. Selon Moignet (ibid.), le groupe du verbe se compose du participe et de l'infinitif. Traditionnellement, le nom et l'adjectif se rassemblent dans le groupe du substantif (Lyon, 1970 : 12). L'adjectif et le participe passé affectent souvent un nom ou un pronom, autrement dit, ils 
fonctionnent toujours dans l'énoncé en tant que complément attributif ou épithète, ils ne peuvent jamais avoir d'impact au niveau de l'action. Lorsque l'injonction est une action demandant une autre action, les énoncés adjectivaux (adjectif et participe passé) sont systématiquement exclus. Quant à l'infinitif et le participe présent, je n'ai en rencontré aucune utilisation; ils ne seront donc pas pris en compte. Dans la troisième section, j'examinerai donc trois types d'énoncés averbaux: les nominaux (pronominaux y compris), les adverbiaux et les prépositionnels. J'étudierai leur répartition dans différents corpus dans la quatrième section.

Pour les énoncés de deux éléments et plus, le classement est fait en fonction du premier élément et je considère cet élément comme le plus important car c'est lui sur lequel le locuteur insiste le plus en le plaçant au début de l'énoncé. Pour les énoncés comme « (...) allez vas-y Jason bien fort » (Corpus de Gomila), «bien fort » peut aller avec « vas-y » ou être indépendant, faute de pause remarquable entre les éléments. Je préfère le considérer comme un énoncé indépendant et je le traite comme une injonction averbale. Tous les exemples non verbaux ont donc étés retenus dès lorsqu'ils étaient interprétables comme des injonctions et ils sont alors classés en fonction de la catégorie « tête ».

\section{Méthodes et corpus d'étude}

L'objectif de cet article est d'étudier les usages des formes averbales qui expriment une injonction, ainsi que d'en comprendre la répartition en fonction de différents genres de discours. Il est intéressant de quantifier des formes averbales qui servent à enjoindre dans des situations variées. La description concerne la caractérisation des injonctifs dans des situations réelles (c'est-à-dire dans les productions orales qui sont rassemblées dans les corpus attestés) ; autrement dit, il s'agit de voir comment les locuteurs utilisent l'injonctif averbal à l'oral, dans tel ou tel genre, et de voir quel emploi est le plus fréquent dans les performances. L'analyse linguistique de ces énoncés doit également rendre compte des phénomènes considérés comme non-standards: les structures non prévues par les grammaires, c'est-à-dire les séquences d'éléments linguistiques ou extralinguistiques «non modélisées». Une construction de l'identité de l'injonction averbale en langue permet de rendre compte des énoncés concrets dans lesquels elle apparaît.

17 Je présente ci-après les situations dans lesquelles les échanges qui constituent mon corpus se déroulent.

Corpus CFPP2000 - Interview (cfpp2000.univ-paris3.fr/): Ce corpus a été recueilli par Branca-Rosoff, Fleury, Lefeuvre (de l'équipe Clesthia Syled) et par Pires. Il s'agit du français parlé dans Paris et son agglomération. Les auteurs s'en sont tenus à un genre d'interaction spécifique : l'interview, sur un thème particulier (la relation des personnes interrogées à leur quartier et plus largement à Paris ou à leur banlieue). Il y a 562.121 mots au total dans la version de mars 2012 que j'utilise.

Corpus CLAPI - Petit commerce : CLAPI est le Corpus de Langue Parlé en Interaction, qui a été constitué à Lyon par les membres du laboratoire ICAR. Je ne prends en compte que deux sous-corpus dont j'ai obtenu l'accès pour l'intégralité: "TABACCO» (Interactions commerciales - Bureau de Tabac-Presse (TABACCO), J. COSNIER, CLAPI : http://clapi.univlyon2.fr) et "Chez le directeur» (http://clapi.univ-lyon2.fr). Le corpus TABACCO a été recueilli dans un bureau de tabac-presse à Lyon en 1986. Il y a 126 locuteurs dont 120 
clients. Les échanges dans le corpus Chez le directeur se déroulent dans le bureau du directeur de foyer.

Corpus de Gomila - didactique et institutionnel (http://syled.univ-paris3.fr/ressources/ corpus-CDMCL2007/Presentation.html) : Ce corpus se compose de 39 séances de lecture de texte dans différentes classes de cours préparatoires et de grandes sections. Les écoles concernées sont implantées dans l'académie d'Aix-Marseille. Les interlocuteurs sont toujours un maitre ou une maitresse et une classe d'enfants de 5 à 7 ans.

Corpus Informatique et corpus Recette - didactique et amical / familial: Ces deux corpus se composent d'enregistrements collectés par les étudiants de licence de l'Université Sorbonne Nouvelle - Paris 3 qui se sont inscrits dans les cours de Mme Branca-Rosoff. Les informateurs et les enquêteurs sont amis ou camarades. Les corpus sont collectés dans les lieux divers : soit chez l'enquêteur, soit chez l'enquêté, soit à un endroit de travail de l'enquêté. Le thème de l'enquête porte sur ce qui est dans l'intérêt de l'enquêté (logiciel ou recette). Une atmosphère amicale est présente dans presque toutes les enquêtes.

Les dimensions très différentes des corpus font qu'ils donnent des indications sur des tendances et non des résultats définitifs.

\section{Les injonctifs averbaux}

Dans cette section, je présente d'abord les caractéristiques linguistiques et pragmatiques des injonctifs averbaux ainsi que la fréquence particulièrement remarquable de certains emplois s'il y a lieu.

\subsection{Les injonctifs nominaux}

24 Je place dans la catégorie des injonctifs nominaux tous les énoncés averbaux qui dépendent d'un nom ou d'un pronom, autrement dit tous les énoncés qui se regroupent autour d'une tête nominale. Ces énoncés peuvent être à un terme ou à deux termes. Parmi les injonctifs nominaux, la mention de la quantité est très fréquente dans le corpus CLAPI. Il est utilisé à la fois par les clients et par les vendeurs :

\begin{tabular}{|c|l|}
\hline CL42 & $\begin{array}{l}\text { euh:: ça/ (.) euh deux gauloises sans filtre/ (0.7) et deux } \\
\text { gauloises blondes }\end{array}$ \\
\hline CL42 & bleues \\
\hline BEA & alors \\
\hline BEA & vingt quatre quatre vingt/ (Corpus TABACCO - CLAPI) \\
\hline
\end{tabular}

La requête du client porte sur la quantité autant que sur les produits dont il a besoin et pourrait être paraphrasée avec le verbe vouloir : « je voudrais deux gauloises sans filtre et deux gauloises blondes bleues ». La requête de la vendeuse porte sur le montant que le client doit payer et peut être paraphrasé par le verbe vouloir pour insister sur la requête : « je voudrais vingt quatre quatre vingt ». Dans le corpus CLAPI, quatre cinquièmes des 
utilisations des énoncés nominaux (85 occurrences) commencés par un nom de quantité, ils représentent $80 \%$ sur le total de 106 occurrences nominales.

Dans le cas où l'énoncé comporte un déterminant défini, un adjectif possessif ou démonstratif, un pronom démonstratif ou un nom propre, l'objet de l'action demandée est bien déterminé. La réalisation de l'action future pointe sur cet objet. Le locuteur désigne précisément l'objet qu'il veut obtenir :

\begin{tabular}{|c|l|}
\hline CL9 & euh: le mon/de libéra[tion] (Corpus TABACCO - CLAPI) \\
\hline M & $\begin{array}{l}\text { le radis et ce petit mot-là que nous connaissons (Corpus de } \\
\text { Gomila) }\end{array}$ \\
\hline M & de oui celui-là (Corpus de Gomila) \\
\hline
\end{tabular}

Dans le cas où le locuteur est dans un environnement qui comporte de nombreux participants (Corpus de Gomila, par exemple, où toute une classe est présente), l'adjectif possessif à la deuxième personne peut aussi intervenir :

\begin{tabular}{|l|l|}
\hline M & $\begin{array}{l}\text { Alexia tes } \\
\text { (Corpus de Gomila) }\end{array}$ \\
\hline
\end{tabular}

Les injonctifs nominaux peuvent comporter un nom et un adjectif. Dans ce cas l'adjectif joue souvent un rôle locatif, c'est-à-dire qu'il aide à localiser l'objet de l'action demandée, et insiste sur l'idée de différence par rapport à un élément choisi comme référence. On observe la même chose dans les cas où il y a un complément du nom ou une sous-phrase relative :

\begin{tabular}{|c|c|}
\hline M & $\begin{array}{l}\text { laissez-le réfléchir fais tes syllabes on y va première syllabe } \\
\text { (Corpus de Gomila) }\end{array}$ \\
\hline$M$ & $(.)+.\underline{\text { la suite des paroles de la sorcière }(. . .)(\text { Corpus de Gomila) }}$ \\
\hline$M$ & $\begin{array}{l}(. .)+\text { alors le mot qui est juste à côté sur le livre là (...) (Corpus } \\
\text { de Gomila) }\end{array}$ \\
\hline
\end{tabular}

Les utilisations des énoncés nominaux accompagnés d'un adjectif, d'un complément ou d'une sous-phrase relative sont assez fréquentes dans le corpus de Gomila. Parmi 263 occurrences nominales trouvées dans ce corpus, près d'un tiers sont des énoncés nominaux de ce type (84 occurrences, soit $31,94 \%)$. Ils sont liés aux consignes des maîtres qui font faire des exercices en classe aux élèves, ils sont donc très ritualisés.

Si l'énoncé est accompagné d'un participe, en surface il y a deux éléments explicites: l'action demandée et l'objet de cette action. Le locuteur demande à l'allocutaire de faire 
l'action exprimée par le participe. Avec «les doigts bien serrés », la maitresse demande à ses élèves de faire l'action de serrer les doigts :

\begin{tabular}{|l|l|}
\hline M & les doigts bien serrés une seule main suffit $+(.$.$) (Corpus de$ \\
Gomila)
\end{tabular}

Dans les injonctifs nominaux à deux termes, le prédicat est un groupe de mots dont la tête est un nom. L'objet de l'action et le récepteur sont exprimés en surface. Ils ont l'ordre sujet - prédicat. L'ordre inversé prédicat - sujet ("groupe 1 les autres») serait aussi acceptable dans ce cas :

\begin{tabular}{|l|l|}
\hline M & $\begin{array}{l}\text { (..) groupe 1 je vous regarde pas les autres groupe 1 (Corpus } \\
\text { de Gomila) }\end{array}$ \\
\hline
\end{tabular}

Lorsqu'il est accompagné d'un adverbe de manière, le locuteur veut insister sur l'objet de l'action et l'intensité dans la réalisation de cette action (l'adverbe d'intensité affecte toujours l'action que l'on reconstruit) ou sur l'objet de l'action et le moment de réalisation de cette action, exprimé par un adverbe de temps, etc. :

\begin{tabular}{|c|l|}
\hline M & $\begin{array}{l}\text { très bien allez Philippe la troisième phrase bien fort (Corpus } \\
\text { de Gomila) }\end{array}$ \\
\hline M & les lettres maintenant (Corpus de Gomila) \\
\hline
\end{tabular}

L'injonctif nominal peut être suivi d'un groupe prépositionnel. S'il s'agit d'une localisation spatiale, ce deuxième élément affecte l'objet de l'action plutôt que l'action :

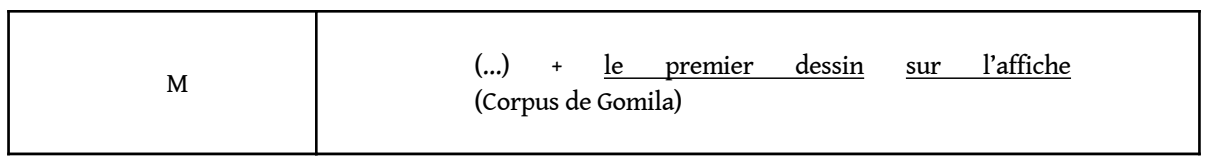

S'il s'agit d'un nom propre ou d'un pronom personnel, souvent suivi de la préposition à, le locuteur désigne directement le(s) récepteur(s). En surface sont exprimés l'objet de l'action demandée et le récepteur. Dans ce cas, le récepteur désigné par le locuteur n'est pas le sujet du verbe reconstitué. L'énoncé ne peut être paraphrasé qu'avec le verbe être: "c'est à vous de lire le groupe 1 » ou « le groupe 1 c'est à vous de lire " pour l'exemple suivant (voir aussi §3.3. de cet article) :

\begin{tabular}{|l|l|}
\hline$M$ & $\begin{array}{l}{[l \ominus] \text { très bien regardez ma langue [lə] groupe } 1 \text { à vous (Corpus }} \\
\text { de Gomila) }\end{array}$ \\
\hline
\end{tabular}


Les injonctifs nominaux à trois éléments sont très rares. J'ai trouvé une seule occurrence dans l'ensemble de mon corpus. Dans cet usage, tous les éléments sont nécessaires : l'objet, le récepteur et l'action demandée pour une bonne réalisation de l'action future sont exprimés en surface, on ne doit pas recourir au contexte linguistique et situationnel pour reconnaître l'action demandée et le récepteur :

\begin{tabular}{|l|l|}
\hline$M$ & $\begin{array}{l}\text { (..) + paragraphe suivant à vous de lire } \\
\text { (Corpus de Gomila) }\end{array}$ \\
\hline
\end{tabular}

\subsection{Les injonctifs adverbiaux}

En principe, tout adverbe peut constituer un énoncé averbal injonctif car il affecte le verbe / l'action de telle ou telle façon selon la signification: spatiale, temporelle, de manière, etc. Les injonctifs adverbiaux supposent un verbe / une action sous-jacent(e), une action déjà faite ou prévue et insuffisamment réussie. Ce qui les distingue des autres c'est qu'en les utilisant, le locuteur veut que l'allocutaire (re)fasse l'action de façon indiquée par l'adverbe. Cette façon de réaliser l'action demandée est particulièrement insistante :

\begin{tabular}{|l|l|}
\hline M & $\begin{array}{l}\text { (..) on continue allez Laura }+ \text { bien fort } \\
\text { (Corpus de Gomila) }\end{array}$ \\
\hline
\end{tabular}

Dans le cas où il y a deux adverbes utilisés, la réalisation de l'action demandée doit satisfaire en même temps les aspects que les deux adverbes expriment :

\begin{tabular}{|l|l|}
\hline M & $\begin{array}{l}\text { on le redit }+ \text { bien fort ensemble } \\
\text { (Corpus de Gomila) }\end{array}$ \\
\hline
\end{tabular}

La manière demandée de réaliser l'action future peut être également comparée à une autre. Dans ce cas, l'énoncé renvoie à la manière dont l'action a été réalisée précédemment à cette action. Le locuteur demande à l'allocutaire de faire la même action mais d'une autre façon que la précédente, indiquée par l'adverbe :

\begin{tabular}{|c|c|}
\hline M & plus fort \\
\hline Gaïs & Ciseaux \\
\hline M & plus fort (Corpus de Gomila) \\
\hline
\end{tabular}

39 Lorsque l'injonctif adverbial est suivi par un groupe nominal ou un groupe prépositionnel, l'insistance porte sur tous ces deux éléments : 


\begin{tabular}{|c|l|}
\hline$M$ & $\begin{array}{l}\text { (..) Théo bien fort deuxième paragraphe tu commences (...) } \\
\text { (Corpus de Gomila) }\end{array}$ \\
\hline$M$ & $\begin{array}{l}\text { allez ensuite à la première image à la première image qu'est ce } \\
\text { qu'on va pouvoir lire alors? Clémence clémence (Corpus de } \\
\text { Gomila) }\end{array}$ \\
\hline
\end{tabular}

\subsection{Les injonctifs prépositionnels}

En général, tout groupe prépositionnel peut constituer un injonctif averbal. Les prépositions que j'ai rencontrées le plus souvent dans mon corpus sont à, avec, en, après, sur. Syntaxiquement, les groupes prépositionnels peuvent jouer le rôle d'un complément d'objet indirect ou de circonstance dans la structure complète. C'est pourquoi Le Goffic (1993 : 519) les appelle «locatifs ». Il s'agit de la localisation spatiale, de la localisation notionnelle, etc. :

\begin{tabular}{|c|l|}
\hline $\mathrm{M}$ & $\begin{array}{l}\text { très bien Pierre à côté le dessin qui est à côté du saladier (...) } \\
\text { (Corpus de Gomila) }\end{array}$ \\
\hline BEA & et voilà au boulot/ (Corpus TABACCO - CLAPI) \\
\hline
\end{tabular}

41 La plupart des utilisations du groupe prépositionnel à que j'ai rencontrées comportent un nom propre ou un pronom personnel. Le Goffic pense que ces types d'énoncés « ont un statut souvent difficile à déterminer; ils correspondent à des actes de langage très divers ", de plus «ils peuvent aussi bien représenter une phrase tronquée qu'une phrase "nominale" la localisation ne peut donc pas (...) se restreindre à être " (1993: 519). Lefeuvre répertorie la valeur d'actance (2003: 249). De notre point de vue, dans la perspective injonctive, en utilisant le groupe prépositionnel $\grave{a}+$ un nom propre ou un pronom personnel, le locuteur désigne explicitement la personne qui doit réaliser l'action future :

\begin{tabular}{|l|l|}
\hline $\mathrm{M}$ & $\begin{array}{l}\text { Bran-don il est écrit mais on ne l'entend pas vas-y à toi (Corpus } \\
\text { de Gomila) }\end{array}$ \\
\hline
\end{tabular}

«à toi » équivaut à « le tour est à toi » ou à « c'est à toi de dire ton prénom ». La personne désignée par le nom propre ou le pronom n'a donc pas le statut de sujet du verbe dans la construction complète mais le statut de récepteur de l'action demandée. La paraphrase correspond plutôt à la forme indirecte avec être qu'à la forme avec un verbe illocutoire.

Dans le cas où l'injonctif prépositionnel est accompagné d'un autre groupe prépositionnel, ce groupe complète le groupe prépositionnel qui précède, il précise la localisation spatiale ou temporelle : 


\begin{tabular}{|c|l|}
\hline$M$ & $\begin{array}{l}\text { (..) non sur la table à côté là } \\
\text { (Corpus de Gomila) }\end{array}$ \\
\hline$M$ & dans le texte pour l'instant (Corpus de Gomila) \\
\hline
\end{tabular}

(...) ah ah ben je sais moi à vous de me le dire on va voir (Corpus de Gomila)

Lorsque le premier groupe comporte un nom propre ou un pronom personnel et la préposition à, qui se combine avec un infinitif autour de la tête de, le récepteur et l'action future sont explicités :

\begin{tabular}{|l|l|}
\hline$M$ & $\begin{array}{l}\text { (...) ah ah ben je sais moi à vous de me le dire on va voir } \\
\text { (Corpus de Gomila) }\end{array}$ \\
\hline
\end{tabular}

Lorsqu'il est accompagné d'un groupe nominal et d'un groupe prépositionnel, l'insistance porte sur le récepteur, l'objet de l'action et la localisation de cet objet :

\begin{tabular}{|l|l|}
\hline M & $\begin{array}{l}\text { (..) d'accord à toi Julien un concours de danse dans une phrase } \\
\text { (Corpus de Gomila) }\end{array}$ \\
\hline
\end{tabular}

\section{Récapitulatifs}

Je présente les fréquences d'utilisation et les comparaisons à l'intérieur d'un sous-corpus dans la première sous-section et celles entre les sous-corpus dans la dernière soussection. Ces fréquences permettent de voir la mobilisation de ces formes averbales dans différentes situations par les usagers.

\subsection{Comptages dans les sous-corpus}

47 Je n'ai rencontré que 10 occurrences des injonctifs averbaux dans le corpus CFPP2000, toutes affirmatives (8 occurrences nominales et 2 occurrences prépositionnelles). De même dans le corpus Informatique, il y a seulement 10 occurrences des injonctifs averbaux (7 occurrences nominales et 3 occurrences prépositionnelles). Par conséquent, les fréquences d'utilisation des injonctifs averbaux dans ces deux corpus ne sont pas prises en considération ici. Je présente ci-dessous les tableaux des utilisations des injonctifs averbaux dans les corpus CLAPI, de Gomila et Recette.

Dans le corpus de Gomila, il y a 419 occurrences d'injonctifs averbaux. Le tableau 1 récapitule les utilisations des injonctifs averbaux dans ce corpus sur l'effectif total de 419. Tableau 1 : les utilisations des injonctifs averbaux dans le corpus de Gomila

\begin{tabular}{|l|l|l|l|l|l|l|l|l|}
\hline Formes & nominales & $\%$ & adverbiales & $\%$ & prépositionnelles & $\%$ & total & $\%$ \\
\hline affirmatives & 259 & 61,81 & 112 & 26,73 & 40 & 9,55 & 411 & 98,09 \\
\hline
\end{tabular}




\begin{tabular}{|l|l|l|l|l|l|l|l|l|}
\hline négatives & 4 & 0,96 & 4 & 0,95 & 0 & 0 & 8 & 1,91 \\
\hline sous-total & 263 & 62,77 & 116 & 27,68 & 40 & 9,55 & 419 & 100 \\
\hline
\end{tabular}

Les utilisations négatives ne représentent que 1,91\% (soit 8 occurrences dont 4 occurrences nominales et 4 occurrences adverbiales (soit $0,89 \%$ pour chacune)). Les utilisations affirmatives représentent 98,09\% (411 occurrences), elles sont les plus fréquentes à toutes les formes. Il n'y a aucune utilisation négative pour l'injonctif prépositionnel.

Les injonctifs nominaux sont les plus fréquemment utilisés (263 occurrences, soit 62,77\%). Les utilisations des injonctifs adverbiaux représentent 27,68\% (116 occurrences). Les utilisations des injonctifs prépositionnels sont les moins fréquentes (40 occurrences, soit 9,55\%).

51 Le tableau 2 récapitule les utilisations des injonctifs averbaux dans le corpus CLAPI. Il y a 112 occurrences d'injonctifs averbaux. Les fréquences sont les pourcentages calculés sur l'effectif total de 112.

Tableau 2 : les utilisations des injonctifs averbaux dans le corpus CLAPI

\begin{tabular}{|l|l|l|l|l|l|l|l|l|}
\hline Formes & nominales & $\%$ & prépositionnelles & $\%$ & adverbiales & $\%$ & total & $\%$ \\
\hline affirmatives & 105 & 93,75 & 4 & 3,57 & 2 & 1,79 & 111 & 99,11 \\
\hline négatives & 1 & 0,89 & 0 & 0 & 0 & 0 & 1 & 0,89 \\
\hline sous-total & 106 & 94,64 & 4 & 3,57 & 2 & 1,79 & 112 & 100 \\
\hline
\end{tabular}

Les utilisations négatives ne représentent que $0,89 \%$ ( 1 occurrence qui est nominale). Les utilisations affirmatives sont écrasantes (111 occurrences, soit 99,11\%), elles sont également les plus fréquentes à toutes les formes.

Les utilisations des injonctifs nominaux sont les plus fréquentes (106 occurrences, soit 94,64\%). Les utilisations des injonctifs adverbiaux sont les moins fréquentes (2 occurrences, soit 1,79\%). Les utilisations des injonctifs prépositionnels sont plus fréquentes que celles des injonctifs adverbiaux mais avec un taux remarquablement bas (4 occurrences, soit 3,57\%).

54 Le tableau 3 présente les utilisations des injonctifs averbaux dans le corpus Recette. Il y a 13 occurrences d'injonctifs averbaux. Les fréquences sont les pourcentages calculés sur l'effectif total de 13.

Tableau 3 : les utilisations des injonctifs averbaux dans le corpus Recette

\begin{tabular}{|l|l|l|l|l|l|l|l|l|}
\hline Formes & nominales & $\%$ & adverbiales & $\%$ & prépositionnelles & $\%$ & total & $\%$ \\
\hline affirmatives & 6 & 46,15 & 1 & 7,692 & 3 & 23,08 & 10 & 76,92 \\
\hline négatives & 0 & 0 & 3 & 23,08 & 0 & 0 & 3 & 23,08 \\
\hline total & 6 & 46,15 & 4 & 30,77 & 3 & 23,08 & 13 & 100 \\
\hline
\end{tabular}


55

utilisations négatives représentent 23,08\% (3 occurrences qui sont toutes adverbiales)

Les utilisations affirmatives représentent $76,92 \%$ (10 occurrences), elles sont les plus fréquentes. Il n'y a pas d'utilisation négative pour les injonctifs nominal et prépositionnel. Les utilisations des injonctifs nominaux sont les plus fréquentes (6 occurrences, soit $46,15 \%)$. Les utilisations des injonctifs prépositionnels sont les moins fréquentes (3 occurrences, soit 23,08\%).

\subsection{Récapitulatifs généraux}

Le tableau 4 présente les utilisations des injonctifs averbaux dans les sous-corpus. Les pourcentages sont calculés sur le nombre total des occurrences des injonctifs averbaux trouvés dans chaque sous-corpus.

Tableau 4 : les utilisations des injonctifs averbaux dans les 5 sous-corpus

\begin{tabular}{|c|c|c|c|c|c|c|c|c|c|c|c|}
\hline \multirow{2}{*}{\multicolumn{2}{|c|}{$\begin{array}{l}\text { Corpus } \\
\text { Formes }\end{array}$}} & \multicolumn{2}{|c|}{ Gomila } & \multicolumn{2}{|c|}{ CLAPI } & \multicolumn{2}{|c|}{ Recette } & \multicolumn{2}{|c|}{ CFPP2000 } & \multicolumn{2}{|c|}{ Informatique } \\
\hline & & eff. & $\%$ & eff. & $\%$ & eff. & $\%$ & eff. & $\%$ & eff. & $\%$ \\
\hline \multicolumn{2}{|l|}{ nominales } & 263 & 62,77 & 106 & 94,64 & 6 & 46,15 & 8 & 80 & 7 & 70 \\
\hline \multicolumn{2}{|c|}{ adverbiales } & 116 & 27,68 & 2 & 1,79 & 4 & 30,77 & 0 & 0 & 0 & 0 \\
\hline \multicolumn{2}{|c|}{ prépositionnelles } & 40 & 9,55 & 4 & 3,57 & 3 & 23,08 & 2 & 20 & 3 & 30 \\
\hline \multirow{2}{*}{ sous-total } & aff. & 411 & 98,09 & 111 & 99,11 & 10 & 76,92 & 10 & 100 & 8 & 80 \\
\hline & nég. & 8 & 1,91 & 1 & 0,89 & 3 & 23,08 & 0 & 0 & 2 & 20 \\
\hline \multicolumn{2}{|l|}{ total } & 419 & 100 & 112 & 100 & 13 & 100 & 10 & 100 & 10 & 100 \\
\hline
\end{tabular}

utilisations négatives sont les moins usuelles dans les sous-corpus. Cependant, il y a une grande différence entre les utilisations négatives dans les sous-corpus : les taux très faibles sont remarqués dans les corpus CFPP2000 (0\%), CLAPI $(0,89 \%)$ et de Gomila $(1,91 \%$ ) ; en revanche ces taux sont considérablement élevés dans les corpus Recette $(23,08 \%)$ et Informatique (20\%). Aucune utilisation négative des injonctifs prépositionnels n'est trouvée dans l'ensemble de mon corpus, de même pour les injonctifs nominaux dans le corpus Recette et pour les injonctifs adverbiaux dans les corpus CLAPI et Informatique.

Les utilisations affirmatives sont donc les plus fréquentes dans tous les sous-corpus, elles varient de 76,92\% dans le corpus Recette à 100\% dans le corpus CFPP2000 ainsi que les utilisations des injonctifs nominaux. Il y a pourtant une grande différence entre les utilisations des injonctifs nominaux dans le corpus CLAPI (94,64\%; les plus élevées) et celles dans le corpus Recette (46,15\% ; les plus basses). La différence est aussi marquée pour les utilisations des injonctifs adverbiaux dans les corpus Recette $(30,77 \%)$ et de Gomila (27,68\%) contre aucune utilisation dans les corpus CFPP2000 et Informatique ou le taux très faible dans le corpus CLAPI $(1,79 \%)$.

Les utilisations des injonctifs prépositionnels sont les moins fréquentes dans les corpus de Gomila et Recette, mais elles sont plus fréquentes que celles des injonctifs adverbiaux dans les corpus CLAPI, CFPP2000 et Informatique. 
Le tableau 5 présente les tendances d'utilisation des injonctifs averbaux dans les souscorpus. Les fréquences sont les pourcentages calculés sur le nombre de mots constituant chaque sous-corpus. Il y a 564 occurrences des injonctifs averbaux trouvées dans l'ensemble de mon corpus, soit 0,07858\% en moyenne sur 717.773 mots au total.

Tableau 5 : Les tendances d'utilisation des injonctifs averbaux dans les 5 sous-corpus

\begin{tabular}{|l|l|l|l|l|l|l|l|l|l|l|}
\hline \multirow{2}{*}{ Corpus } & \multirow{2}{*}{$\begin{array}{l}\text { nb. } \\
\text { mots }\end{array}$} & & \multicolumn{2}{l}{ nominaux } & \multicolumn{2}{l|}{ adverbiaux } & \multicolumn{2}{l|}{ prépositionnels } & \multicolumn{2}{l}{ total } \\
\cline { 3 - 12 } & eff. & fréquences & eff. & fréquences & eff. & fréquences & eff. & fréquences \\
\hline Gomila & 97.879 & 263 & 0,26860 & 116 & 0,11851 & 40 & 0,04087 & 419 & 0,42808 \\
\hline CLAPI & 14.757 & 106 & 0,71830 & 2 & 0,01355 & 4 & 0,02711 & 112 & 0,75896 \\
\hline Recette & 7.067 & 6 & 0,08490 & 4 & 0,05660 & 3 & 0,04245 & 13 & 0,18395 \\
\hline CFPP2000 & 562.121 & 8 & 0,00142 & 0 & 0 & 2 & 0,00036 & 10 & 0,00178 \\
\hline Informatique & 35.949 & 7 & 0,01947 & 0 & 0 & 3 & 0,00835 & 10 & 0,02782 \\
\hline Total & 717.773 & 390 & 0,05434 & 122 & 0,01690 & 52 & 0,00724 & 564 & 0,07858 \\
\hline
\end{tabular}

61 Les sous-corpus sont placés selon le nombre total décroissant d'importance des occurrences d'injonctifs averbaux trouvées dans chaque sous-corpus. Le corpus de Gomila est placé au premier rang, suivi par les corpus CLAPI, Recette. Les corpus CFPP2000 et Informatique se placent au dernier rang.

62 Les tendances d'utilisation des formes injonctives nominales varient de manière très importante selon les sous-corpus. Pour les injonctifs nominaux, les locuteurs du corpus CFPP2000 sont ceux qui les utilisent le moins $(0,00142$ occurrences sur cent mots du corpus). Placée au dernier rang de quantité, la tendance d'utilisation des injonctifs nominaux dans le corpus Recette est remarquablement élevée (0,08490 occurrences sur cent mots du corpus), elle est donc placée au troisième rang de densité. Placé au premier rang en termes de fréquence absolue (à cause de la quantité de mots qui constituent le corpus), le corpus de Gomila ne se place qu'au deuxième rang de densité $(0,26860$ occurrences sur cent mots du corpus). La tendance d'utilisation des injonctifs nominaux est particulièrement élevée dans le corpus CLAPI $(0,71830$ occurrences sur cent mots du corpus), elle est donc 2,67 fois plus élevée que dans le corpus de Gomila ; 8,46 fois plus élevée que dans le corpus Recette; 36,89 fois plus élevée que dans le corpus Informatique; 504,72 fois plus élevée que dans le corpus CFPP2000 et 13,22 fois plus élevée que la tendance moyenne.

La forte densité des utilisations des injonctifs nominaux dans le corpus CLAPI est liée au genre d'activités évoquées dans ce corpus. Le corpus CLAPI a des affinités avec le petit commerce, la transaction s'effectue en face à face entre le vendeur et le client. La requête du produit est souvent faite par un énoncé nominal, la routinisation est donc très forte. Sur 122 échanges entre les vendeurs et les clients du corpus CLAPI, il y a 106 énoncés injonctifs nominaux. Autrement dit, il y a à peu près un injonctif nominal $(0,86885)$ par échange en moyenne. Cette forte densité des utilisations des injonctifs nominaux dans ce 
corpus peut être expliquée aussi par la contrainte de temps, la durée de chaque échange étant plus ou moins d'une minute (130 minutes/122 échanges).

Pour les injonctifs prépositionnels, les locuteurs du corpus Recette sont ceux qui les utilisent le plus, (0,10165 occurrences pour cent mots du corpus). Cependant, il n'y a pas de grande différence entre la tendance d'utilisation des injonctifs prépositionnels dans ce corpus et celle dans le corpus de Gomila, elle n'est que de 1,04 fois plus élevée que dans le corpus de Gomila ; 1,57 fois plus élevée que dans le corpus CLAPI ; 5,09 fois plus élevée que dans le corpus Informatique ; 119,31 fois plus élevée que dans le corpus CFPP2000 et 5,86 fois plus élevée que la tendance moyenne.

Pour les injonctifs adverbiaux, la tendance d'utilisation est la plus élevée dans le corpus de Gomila ( 0,11851 occurrences pour cent mots du corpus). Les injonctifs adverbiaux sont les moins utilisés ( 0 occurrences) dans les corpus CFPP2000 et Informatique. La tendance d'utilisation des injonctifs adverbiaux dans le corpus de Gomila est 2,09 fois plus élevée que dans le corpus Recette ; 8,74 fois plus élevée que dans le corpus CLAPI et 6,97 fois plus élevée que la tendance moyenne.

Pour les injonctifs averbaux en général, la tendance d'utilisation dans le corpus CFPP2000 est la moins élevée ( 0,00178 sur cent mots du corpus). Cette tendance est la plus élevée dans le corpus CLAPI ( 0,75896 pour cent mots du corpus), elle est 1,77 fois plus élevée que dans le corpus Gomila ; 4,13 fois plus élevée que dans le corpus Recette ; 27,28 fois plus élevée que dans le corpus Informatique; 426,63 fois plus élevée que dans le corpus CFPP2000 et 9,66 fois plus élevée que la tendance moyenne.

La forte densité des utilisations des injonctifs adverbiaux dans le corpus de Gomila est caractérisée par le genre d'activités évoquées dans ce corpus (enseignement apprentissage de lecture). Presque tous les injonctifs adverbiaux sont utilisés par les maitres et les maîtresses. Ils ordonnent aux élèves de faire l'action future (souvent de locution) selon la façon indiquée par l'adverbe. L'énoncé précédent ou le contexte permet de reconnaître l'action demandée (lire, dire, etc.).

Il y a une forte baisse des utilisations des injonctifs adverbiaux par rapport à celles des injonctifs nominaux et prépositionnels dans le corpus CLAPI. La tendance d'utilisation des injonctifs adverbiaux est 53 fois moins élevée que celle des injonctifs nominaux et 2 fois moins élevée que celle des injonctifs prépositionnels dans ce corpus. Cela résulte de l'activité évoquée dans le corpus (transaction commerciale) et de la nuance injonctive que l'énoncé adverbial peut exprimer (la façon de réaliser l'action demandée). L'injonction la plus souvent utilisée dans ce corpus est la requête du produit et de l'argent, l'action demandée impliquée étant de donner soit le produit soit de l'argent. Partant, une injonction qui insiste sur la façon de réaliser l'action ou sur la localisation semble inutile dans cette situation.

Les injonctifs averbaux sont nombreux dans le corpus CLAPI parce qu'il est lié au contexte des échanges commerciaux, ils sont utilisés pour la requête soit du produit soit de l'argent. Ce qui attire l'attention des locuteurs dans ce corpus est le produit pour le client et l'argent pour le vendeur. L'efficacité l'emporte. À certains moments, les locuteurs n'ont même pas de temps pour échanger quelques mots autres que ces requêtes.

Les injonctifs averbaux apparaissent avec une intensité remarquable dans le corpus CLAPI : 0,91803 fois sur un échange. Avec une durée de plus ou moins une minute en moyenne pour chaque échange, ce moyen parait le meilleur choix. 


\section{Conclusion}

71 La présence du verbe joue un rôle important pour la reconnaissance du récepteur et de l'action future que le locuteur veut faire réaliser. La reconnaissance du récepteur et de l'action demandée est plus difficile pour l'injonctif averbal, surtout lorsque l'énoncé compose un seul élément (un nom, un adverbe, etc.). Le recours au contexte linguistique et situationnel permet de les reconnaitre et de reconstituer la structure complète, surtout le verbe/l'action.

Les injonctifs averbaux peuvent être considérés comme les moyens injonctifs les plus directs car la forme la plus proche de cette forme averbale est impérative, seuls les éléments sur lesquels insiste le locuteur sont exprimés en surface, les autres sont éliminés. L'attitude, la volonté et l'orientation du locuteur sont nettement exprimées dans l'énoncé bien qu'il n'y en ait aucun indice en surface.

73 Les injonctifs averbaux sont plus fréquemment utilisés dans les situations où la relation est très hiérarchisée entre les interlocuteurs (enseignant / adulte - enseigné / enfant) ou bien où le principe de politesse cède la place à un principe de célérité (au bureau de tabac en cas d'affluence). La forte densité des utilisations des injonctifs averbaux dans les corpus CLAPI et de Gomila en témoigne. Lorsque la relation entre les interlocuteurs est plus égalitaire et que l'activité dans laquelle ils sont engagés est moins routinisée et n'implique pas une forte contrainte de temps les injonctifs averbaux sont moins nombreux comme dans les corpus Informatique et Recette.

74 L'injonctif averbal est souvent utilisé en comparaison avec une action prévue ou insuffisamment réussie. Les utilisations de cette forme injonctive averbale demandent un "coût cognitif» plus élevé que d'autres formes injonctives verbales. Cela explique pourquoi il apparaît plus dans les situations très routinisées et de fortes contraintes que dans les autres.

Le corpus d'entretiens CFPP2000 est peu propice à l'injonction averbale (10 occurrences, soit 0,00178 occurrences sur cent mots du corpus). L'objectif de ces entretiens est de faire parler, raconter des anecdotes, des histoires qui se sont passées au lieu d'habitation de l'enquêté(e). Il apparaît que la plupart des injonctions, liées à la régulation des interactions, sont des routines conversationnelles (attendez, écoutez, hein...).

\section{BIBLIOGRAPHIE}

BALLY, C. (1944), Linguistique générale et linguistique française, Berne : Francke.

BenVEniste, E. (1966), Problèmes de linguistique générale I, Paris : Gallimard.

Bilger M. \& CAPPEAU P. (2003), « Ce que les corpus nous apprennent sur la langue », actes du colloque « Langue et étude de la langue », (5-7 juin 2003, IUFM de Marseille). 
BILGER M. \& CAPPEAU P. (2004), «L'oral ou la multiplication des styles ». Langage et société, 2004/3 - n 109, p. 13 à 30.

BLANCHE-BENVENISTE, C. (1997), Approches de la langue parlée, Paris : Ophrys.

CAPPEAU P. et GADET F. (2007a), L'exploitation sociolinguistique des grands corpus. Maître-mot et pierre philosophale. Revue française de linguistique appliquée, 2007/1 - Vol. XII, p. 99 à 110

CAPPEAU P. et GADET F. (2007b), Où en sont les corpus sur les français parlés ? Revue française de linguistique appliquée, 2007/1 - Vol. XII, p. 129 à 133

DEULOFEU, H.-J. (2004), « Les énoncés non verbaux sont des énoncés comme les autres », La rime et la raison, Hommage à Benoît de Cornulier, Paris : Champion.

HABERT B. (2000), Des corpus représentatifs : de quoi, pour quoi, comment ? in Bilger (éd.), Linguistique sur Corpus. Etudes et réflexions, Cahiers de l'Université de Perpignan, No 31, p. 11-58, 2000.

Habert B., NazARenKo A. et SAlem A. (1997), Les linguistiques de corpus. U Linguistique. Armand Colin/Masson, Paris, 1997.

HJEMSLEV, L. (1971), « Le verbe et la phrase nominale », Essais linguistiques, Paris : Minuit.

JESPERSEN, O. (1924), The Philosophy of Grammar, Londres: George Allen \& Unwin. Trad. fr. : A.-M. LÉONARD (1971), La philosophie de la grammaire, Paris : Minuit.

LE GOFFIC, P. (1993), Grammaire de la phrase française, Paris : Hachette.

LEFEUVRE, F. (2003), La phrase averbale en français, Paris : L'Harmattan.

LYONS, J. (1970), Linguistique générale. Introduction à la linguistique théorique, Paris : Larousse.

MAYAFFRE D. (2005), Rôle et place de linguistique de corpus : réflexion introductive. Actes des Journées d'Etude Toulousaines JETOU 2005, « Rôle et place des corpus en linguistique », Toulouse, 2005, p. 5-17. http://www.revue-texto.net/Corpus/Publications/Mayaffre_Corpus.html.

MAYAFFRE D. (2005), Rôle et place de linguistique de corpus : réflexion introductive. Actes des Journées d'Etude Toulousaines JETOU 2005, « Rôle et place des corpus en linguistique », Toulouse, 2005, p. 5-17. http://www.revue-texto.net/Corpus/Publications/Mayaffre_Corpus.html.

MEILLET, A. (1924), Traité de grammaire comparée des langues indo-européennes, Paris : Champion.

MOIGNET, G. (1981), Sémantique de la langue française, Paris : Klincksieck.

MONDADA L. (2005), L'analyse de corpus en linguistique interactionnelle : de l'étude de cas singuliers à l'étude de collections. In A. Condamines (dir.), Sémantique et corpus, Londres, Hermès.

PERROT, J. (1994), « Eléments pour une typologie des structures informatives ». Mémoires de la Société de Linguistique de Paris, n.s.t. II , 13,-26.

RIEGEL, M., PELLAT, J-C. \& RIOUL, R. (2009), Grammaire méthodique du français, Paris : Presses Universitaires de France, 1ère éd. 1994.

SEARLE, J.R. (1979), Expression and Meaning: Studies in the Theory of Speech Acts, Cambridge: Cambridge University Press.

SEARLE, J.R. (1990), “A Classification of Illocutionary Acts", in D. Carbaugh (ed), Cultural

Communication and Intercultural Contact, 349-372. 
TESNIÈRE, L. (1959), Eléments de syntaxe structurale, Paris : Klincksieck.

WAGNER, R-L. \& PINCHON, J. (1962), Grammaire

\section{RÉSUMÉS}

Cet article présente une analyse des caractéristiques linguistiques et pragmatiques des injonctifs averbaux (sans verbe conjugué) en s'appuyant sur l'analyse des occurrences tirées de 5 souscorpus différents. Il s'agit de voir comment les compétences linguistiques sont mobilisées par les locuteurs pour satisfaire les attentes normatives dans différentes situations sociales.

This article is a linguistic and pragmatic description of non-verbal imperative utterances, basing on the analysis of occurrences withdrawn from five different sub-corpus. This is to know how language skills are mobilized by speakers to satisfy the normative expectations in different social situations.

\section{INDEX}

Mots-clés : injonction, énoncé averbal, oralité, corpus, genres de discours, analyse linguistiquepragmatique, analyse de corpus

Keywords : imperatives, non-verbal utterance, orality, corpus, discourse genres, linguisticpragmatic analysis, corpus analysis

\section{AUTEUR}

\section{CHINH NGUYEN MINH}

Université Sorbonne Nouvelle - Paris 3

SYLED - ED268

nguyenminhchinh_vn@yahoo.com 